\title{
Inverse kinematic control algorithm for a welding robot - positioner system to trace a $3 \mathrm{D}$ complex curve
}

\author{
Chu A My* \\ Faculty of Aerospace Engineering \\ Le Quy Don Technical University \\ 236 Hoang QuocViet, Hanoi, Vietnam \\ mychuanh@yahoo.com \\ Chi Hieu Le \\ Faculty of Science and Engineering, \\ University of Greenwich \\ Central Avenue, Chatham Maritime, Kent \\ ME4 4TB. United Kingdom \\ C.H.Le@gre.ac.uk
}

\author{
Bui H Tung \\ Faculty of Aerospace Engineering \\ Le Quy Don Technical University \\ 236 Hoang QuocViet, Hanoi, Vietnam \\ hoangtungbui@gmail.com
}

\author{
Duong X Bien \\ Advanced Technology Center \\ Le Quy Don Technical University \\ 236 Hoang QuocViet, Hanoi, Vietnam \\ xuanbien82@yahoo.com
}

\begin{abstract}
The welding robots equipped with rotary positioners have been widely used in several manufacturing industries. However, for welding a 3D complex weldseam, a large number of points should be taught to make the weld path smooth. This is a very tedious job and is not an easy way to teach a robot - rotary positioner system since the robot and the positioner must move simultaneously at the same time. Therefore, in this paper, a new inverse kinematics algorithm is proposed to generate the motion codes for a six DOFs welding robot incorporated with a rotary positioner. In the algorithm, the kinematic error between the desired weld path and the calculated weld path is minimized, and the actual welding error is controlled so that it is always less than an allowable limit. It has shown that the proposed algorithm is useful in developing an offline CAD-based programming tool for robots when welding complex 3D paths. The use of the algorithm increases the accuracy of the welding torch positioning and orientation relative to the required welding joints, and reduces the time for teaching a welding robot - positioner system. Simulation scenarios demonstrate the effectiveness of the proposed method.
\end{abstract}

Index Terms - Welding robot, inverse kinematics, robot offline programming, robot with positioner

\section{INTRODUCTION}

In recent years, the planning and generation of the motion codes for a welding robot cooperated with a rotary positioner are crucial in welding 3D complex joints. Automated welding solutions for complex weldseams without human intervention are nearly impossible due to the non-uniform and irregular geometries of the welding grooves. For a given welding task, workers have to use the robot teach pendant to indicate the welding waypoints and the robot path for the robot to follow a welding pass. When welding a 3D freeform curve, a positioner and the robot have to move simultaneously. In this situation, the robot and a positioner are unified to become a redundant robotic system. Teaching such the robot to move relative to and cooperate accurately with the positioner carrying a workpiece is challenging. Therefore, to increase the quality of the welded parts and efficiency of the welding process, CAD-based offline programming methods which take into account the optimal solution of the inverse kinematics for the welding robotic systems is a practical need. The development in CAD-based robot programming paradigm enables a satisfactory robot path to be generated and simulated with the ideal (or nominal) dimensions of a joint and the exact robot kinematics. In welding of complex joints, the CAD features on a joint can be extracted to form the welding groove, which is used to generate the welding passes and the associated robot paths.

In the literature, there has been a number of researches focusing on offline programming and programming optimization for a welding robot with a positioner [1-7]. An analytical solution of the inverse kinematic problem that deals with the explicit definition of the weld joint orientation relative to the gravity was addressed by Pashkevich et al. [1]. The minimization of the positioner motion for an arc welding robot system was studied in [2]. An off-line programming system for a welding robot was developed in [3], where the workpieces and the weldseam were represented by analytical geometries. In particular, the investigation [4] proposed a method to optimize the robot and positioner motions in redundant robotic system for the fiber placement process. Using this method, the user can find time-optimal smooth profiles for the joint variables while taking into account full capacities of the robotic system expressed by the maximum actuated joint velocities and accelerations. The research [5] presented an offline programming approach for optimal robot path planning of the 
centroid pass when welding Y-joints. The optimal process planning for a welding robot with a positioner was well investigated in $[6,7]$.

Although the offline programming methods for a welding robot with a positioner have been addressed in the past, however little attention has been paid to a robust inverse kinematic control algorithm for calculating the motions of the redundant robotic system (a welding robot and a rotary positioner) when welding a $3 \mathrm{D}$ complex curve. Most of the previous methods considered the linear or circular weldseams when computing the motions of the positioner and the robot. In contrast to the previous investigations, this study develops an effective and robust computation law which is originated from our earlier investigation [8] for calculating the joint coordinates of the robot and the rotation of the positioner. The main idea of this research is that, the kinematic modelling of the robot positioner system is treated as the kinematic modelling of a closed kinematic chain of a 5-axis CNC [9-12], a serial parallel robot [13] or a redundant manipulator [14]. In this manner, the robot and the positioner are considered as two collaborative manipulators, one manipulator carrying the welding torch and one manipulator carrying the workpiece. The two manipulators are constrained through the welding path during the processing time. In order to formulate the kinematics model of the welding system, the two kinematic chains of the robot and the positioner are unified as an open redundant serial kinematic chain for the entire robotic system. The first joint of the unified kinematic chain is the revolute joint of the rotary positioner, and the last link of the robotic system is the endeffector - the welding torch. Since the robot - positioner is a redundant and complex robotic system, in this paper, the inverse kinematic solution is numerically searched in the joint space. In particular, the tracking error between the desired welding path and the actual tool path is controlled within an allowable limit. Numerical examples show the robustness and effectiveness of the algorithm developed in this study.

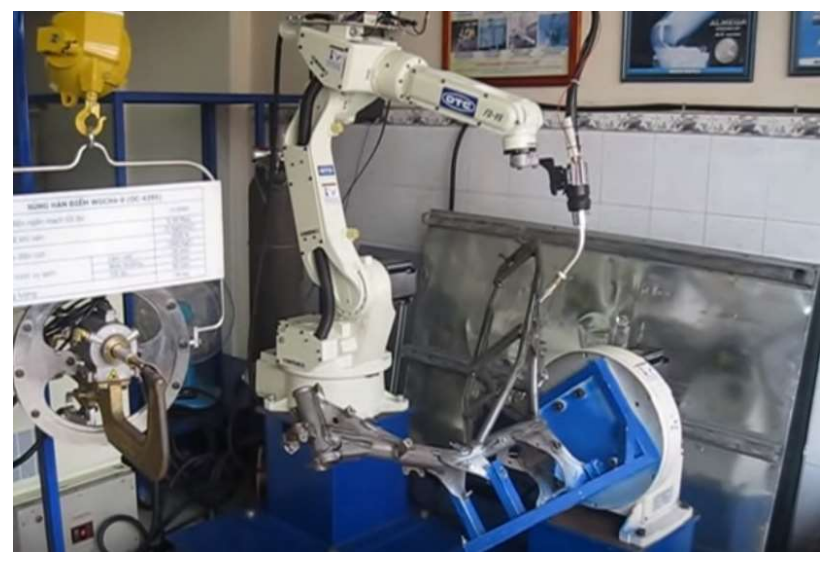

Fig. 1. The welding robot - positioner system FDV8

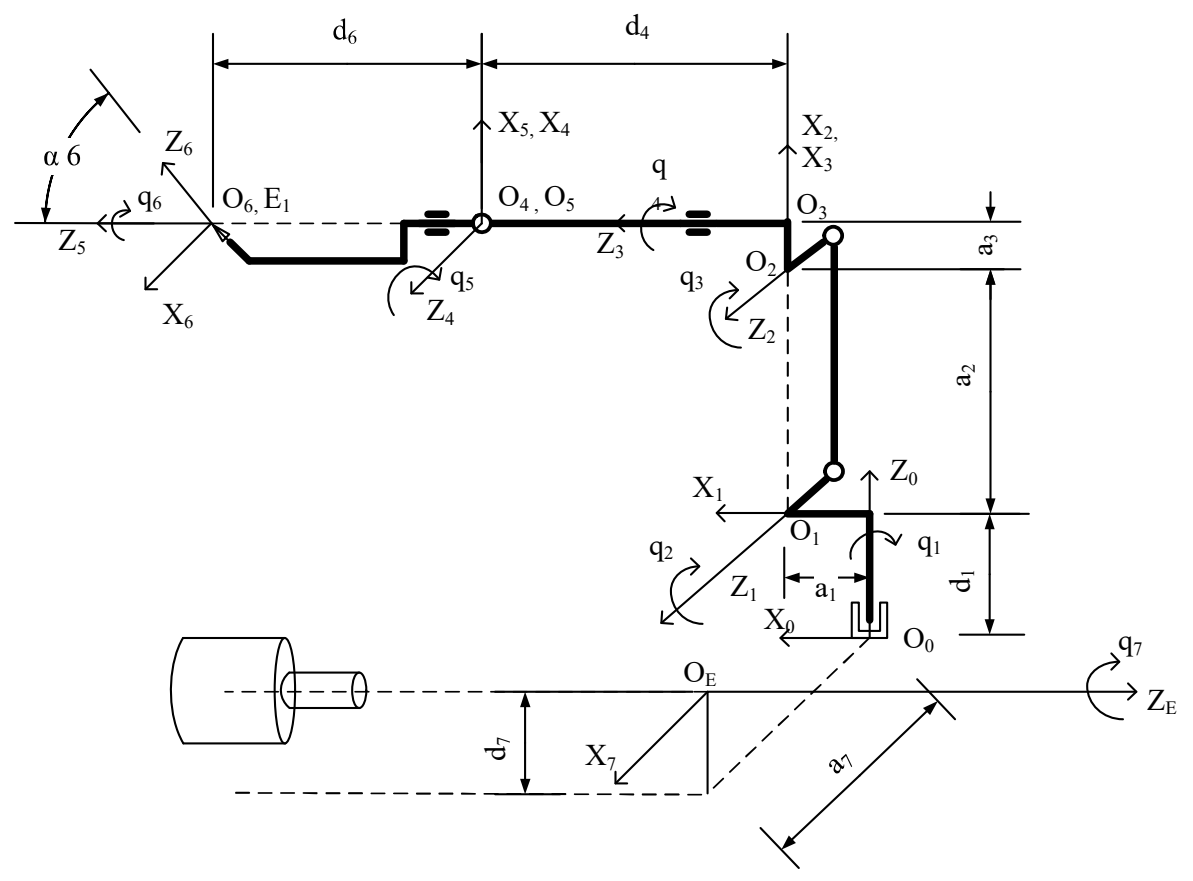

Fig. 2. The kinematic model of the robot - positioner FDV8

\section{KINEMATICS MODELLING AND ROBUST INVERSE KINEMATIC ALGORITHM}

In this section, a welding robot 6-DOFs FD-V8 with a rotary positioner (Fig. 1) are considered, in which the kinematic diagram is shown in Fig. 2. The coordinate systems $(O X Y Z)_{0}$ is the reference frame and is fixed to the base. The frame of the end-effector is $(\mathrm{OXYZ})_{5}$. The frame of swivel table is $(O X Y Z)_{6}$. All of frames are assigned following the DenavitHartenberg (D-H) notation. The parameters of the unified kinematic chain are described in Tab 1. The transformation 
matrices of all frames are determined by homogeneous transformation D-H matrices $\mathbf{H}_{i}^{i-1} ; i=1 \div 7$ which is presented as

$$
\mathbf{H}_{i}^{i-1}=\left[\begin{array}{cccc}
\cos \theta_{i} & -\sin \theta_{i} \cos \alpha_{i} & \sin \theta_{i} \sin \alpha_{i} & a_{i} \cos \theta_{i} \\
\sin \theta_{i} & \cos \theta_{i} \cos \alpha_{i} & -\cos \theta_{i} \sin \alpha_{i} & a \sin \theta_{i} \\
0 & \sin \alpha_{i} & \cos \alpha_{i} & d_{i} \\
0 & 0 & 0 & 1
\end{array}\right]
$$

TABLE 1. KINEMATIC PARAMETERS OF LINKS

\begin{tabular}{|c|c|c|c|c|}
\hline Link & $\theta_{i}$ & $d_{i}$ & $a_{i}$ & $\alpha_{i}$ \\
\hline 1 & $q_{1}$ & $d_{1}$ & $a_{1}$ & $-\frac{\pi}{2}$ \\
\hline 2 & $q_{2}$ & 0 & $a_{2}$ & 0 \\
\hline 3 & $q_{3}$ & 0 & $a_{3}$ & $-\frac{\pi}{2}$ \\
\hline 4 & $q_{4}$ & $d_{1}$ & 0 & $\frac{\pi}{2}$ \\
\hline 5 & $q_{5}$ & 0 & 0 & $-\frac{\pi}{2}$ \\
\hline $6\left(E_{1}\right)$ & $q_{6}$ & $d_{6}$ & 0 & $-\frac{\pi}{4}$ \\
\hline$E$ & $q_{7}$ & $d_{7}$ & $a_{7}$ & $-\frac{\pi}{2}$ \\
\hline
\end{tabular}

The location and direction of the end-effector can be determined as

$$
\mathbf{H}_{7}^{0}=\mathbf{H}_{1}^{0} \mathbf{H}_{2}^{1} \ldots \mathbf{H}_{7}^{6}=\left[\begin{array}{cc}
\mathbf{A}_{n} & \mathbf{r}_{E} \\
0^{T} & 1
\end{array}\right]
$$

Where, $\mathbf{A}_{n}$ is the rotational matrix and $\mathbf{r}_{E}=\mathbf{x}$ is the position vector of the end-effector point.

Assume that the desired path $\mathbf{x}_{d}(t)$ of end-effector in workspace respect to time is given. The goal is to find the value of joint variables in joint space $\mathbf{q}(t), \dot{\mathbf{q}}(t)$ that reproduces the given path. Define the joint variable vector as below

$$
\mathbf{q}=\left[\begin{array}{llll}
q_{1} & q_{2} & \ldots & q_{7}
\end{array}\right]^{T}
$$

The forward kinematic equations can be given as

$$
\mathbf{x}=\mathbf{f}(\mathbf{q})
$$

The differential kinematics equation is described as

$$
\dot{\mathbf{x}}=\mathbf{J}(\mathbf{q}) \dot{\mathbf{q}}
$$

The Jacobian matrix $\mathbf{J}(\mathbf{q})=\frac{\partial \mathbf{f}}{\partial \mathbf{q}}$ is presented as

$$
\mathbf{J}(\mathbf{q})=\left[\begin{array}{llllll}
J_{11} & J_{12} & J_{13} & J_{14} & \ldots . & J_{17} \\
J_{21} & J_{22} & J_{23} & J_{24} & \ldots . & J_{27} \\
J_{31} & J_{32} & J_{33} & J_{34} & \ldots . & J_{37}
\end{array}\right]
$$

The welding robot FDV8 is a redundant system. The inverse kinematic problem for a redundant robot has multiple solutions in general. Due to the non-square Jacobian matrix for 7-DOFs robot, the basic inverse solution to (5) is obtained by using the pseudoinverse $\mathbf{J}^{*}$ of the matrix $\mathbf{J}$ and the inverse solution can then be written as [8]

$$
\dot{\mathbf{q}}=\mathbf{J}^{*}(\mathbf{q}) \dot{\mathbf{x}}
$$

where, the pseudoinverse $\mathbf{J}^{*}$ can be computed as

$$
\mathbf{J}^{*}=\mathbf{J}^{T}\left(\mathbf{J} \mathbf{J}^{T}\right)^{-1}
$$

A common method of including the null space in a solution is the formulation in [8] and the general inverse solution can be described as

$$
\dot{\mathbf{q}}=\mathbf{J}^{*}(\mathbf{q}) \dot{\mathbf{x}}+\left(\mathbf{I}-\mathbf{J}^{*}(\mathbf{q}) \mathbf{J}(\mathbf{q})\right) \dot{\mathbf{q}}_{0}
$$

where, $\mathbf{I}$ is the unit matrix with size $7 \times 7$ and $\mathbf{q}_{0}$ is the initial joint vector.

Because open-loop solutions of joint variables through numerical integration unavoidably lead to errors in workspace. In order to overcome these drawbacks, the closed-loop algorithm is used based on the path error $\mathbf{e}$ in workspace between the desired and actual path. Considering the location error $\mathbf{e}$ and its derivative $\dot{\mathbf{e}}$ which can be given as

$$
\mathbf{e}=\mathbf{x}_{d}-\mathbf{x} ; \dot{\mathbf{e}}=\dot{\mathbf{x}}_{d}-\dot{\mathbf{x}}
$$

The generalized closed-loop inverse kinematic algorithm can be expressed

$$
\dot{\mathbf{q}}=\mathbf{J}^{*}(\mathbf{q})\left(\dot{\mathbf{x}}_{d}+\mathbf{K}_{p}\left(\mathbf{x}_{d}-\mathbf{x}\right)\right)
$$

Combining (5) and (11) together, there will be

$$
\dot{\mathbf{e}}+\mathbf{K}_{p} \mathbf{e}=0
$$

where, $\mathbf{K}_{p}$ is a symmetric positive definite matrix.

Combining (9) and (12) together, the inverse kinematic solution of redundant robot based on closed-loop algorithm is given as

$$
\dot{\mathbf{q}}=\mathbf{J}^{*}(\mathbf{q})\left(\dot{\mathbf{x}}_{d}+\mathbf{K}_{p}\left(\mathbf{x}_{d}-\mathbf{x}\right)\right)+\left(\mathbf{I}-\mathbf{J}^{*}(\mathbf{q}) \mathbf{J}(\mathbf{q})\right) \dot{\mathbf{q}}_{0}
$$

The algorithm is implemented in MATLAB/SIMULINK environment as shown in Fig. 3. 


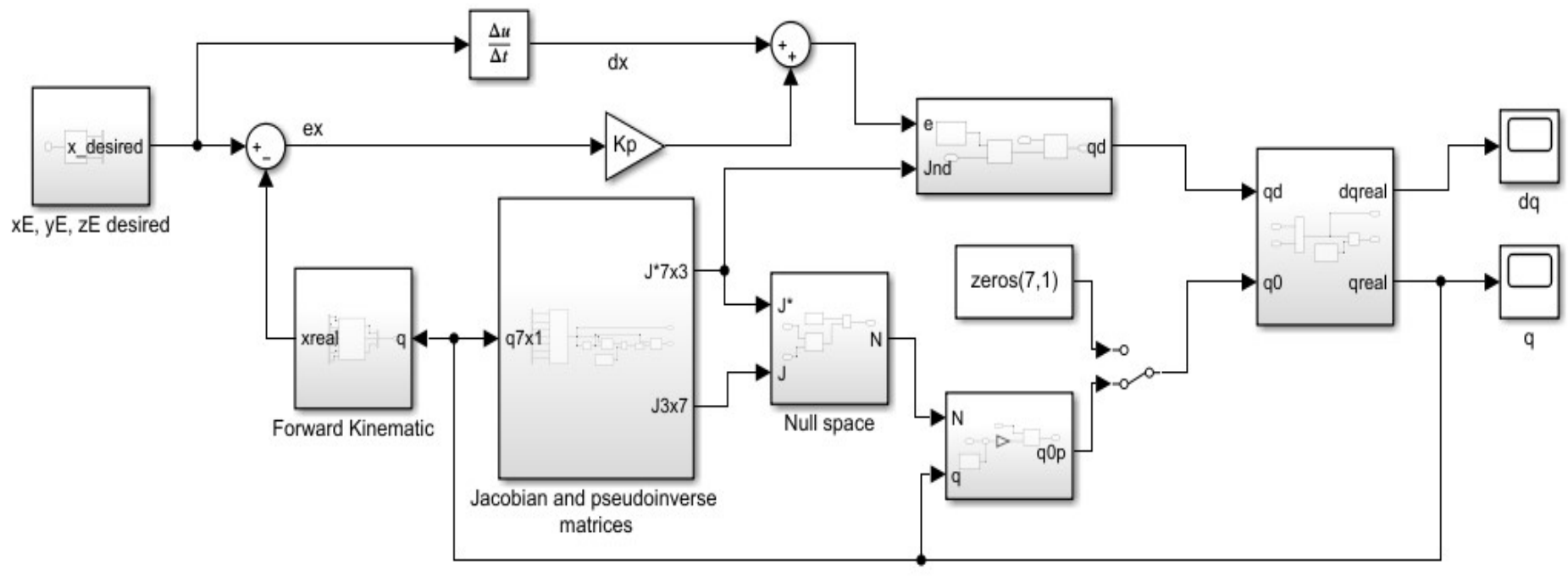

Fig. 3. The inverse kinematic algorithm implemented in SIMULINK

\section{NUMERICAL EXAMPLE AND SIMULATION}

In this section, the inverse kinematic algorithm constructed for the welding robot FDV8 is demonstrated. The desired welding path is given as follows.

$$
\left\{\begin{array}{l}
x_{E}=0.02 * \mathrm{t}+0.01 \quad(\mathrm{~m}) \\
y_{E}=0.3 \sin \left(\frac{\pi}{2} t\right) \quad(\mathrm{m}) \\
z_{E}=0.3 \cos \left(\frac{\pi}{2} t\right) \quad(\mathrm{m})
\end{array}\right.
$$

Note that the tool path (14) shown in Fig. 5 is a complex 3D curve. Teach the robot and the positioner by using the teach pedant to weld this curve is challenging. This is a good example of welding path to demonstrate an offline programming algorithm.

Parameters of the links of the robotic system are as follows. $a_{1}=0.16(m), a_{2}=0.615(m), a_{3}=0.125(m), \mathrm{a}_{5}=0$, $\mathrm{d}_{4}=0.65(\mathrm{~m}), d_{6}=0.475(\mathrm{~m}), \mathrm{d}_{7}=0.45(\mathrm{~m}), \mathrm{K}_{p i}=100$

The desired path and velocity of the end-effector are shown in Figs. 4-6. The simulation results are presented in Figs. 7-10. Fig. 7 shows the joint variable values. It is shown that the displacement value of joint 2, joint 3 and joint 5 are bigger than the other ones. The joint velocities are presented in Fig. 8 while the rotation of the positioner (joint 7) is described in Fig. 9. The errors between the desired and calculated paths are shown in Fig. 10. The maximum value of the error is $0.8 \times 10^{-3}(\mathrm{~m})$.

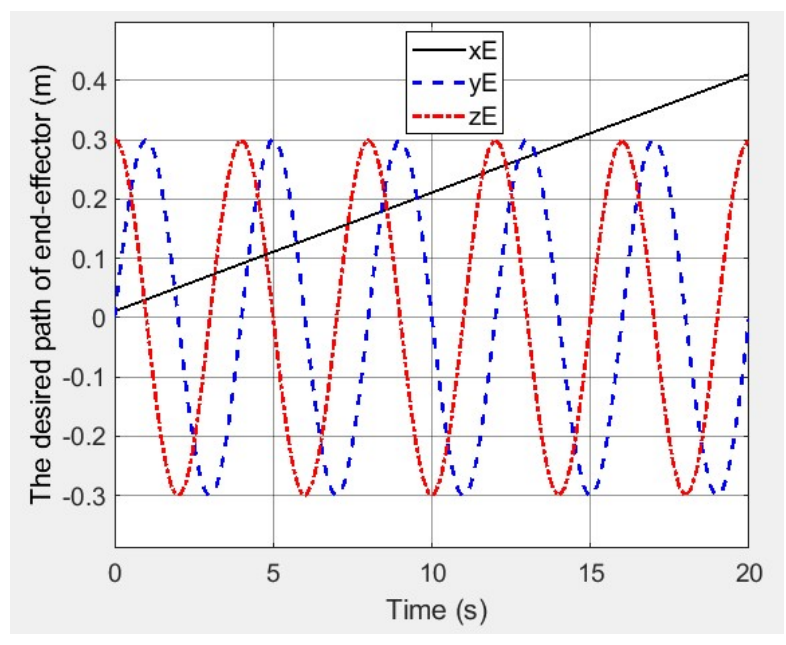

Fig. 4. The desired path of the end-effector

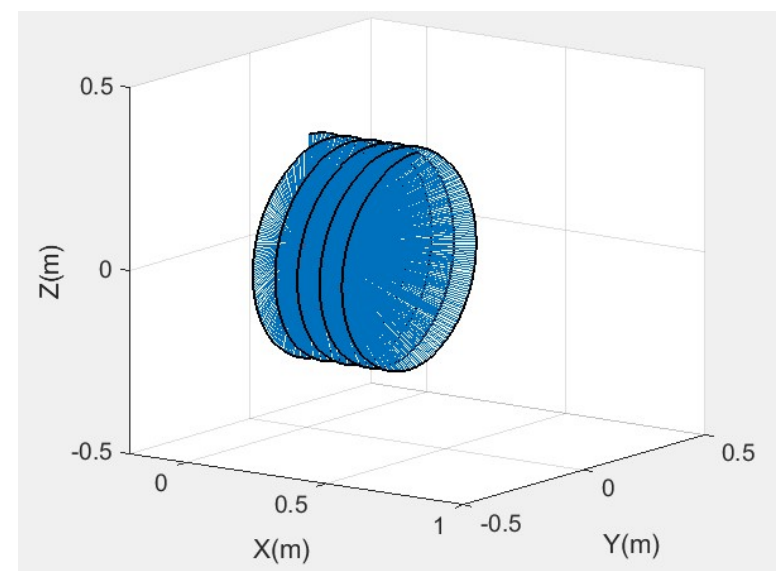

Fig. 5. The desired path in the workspace 


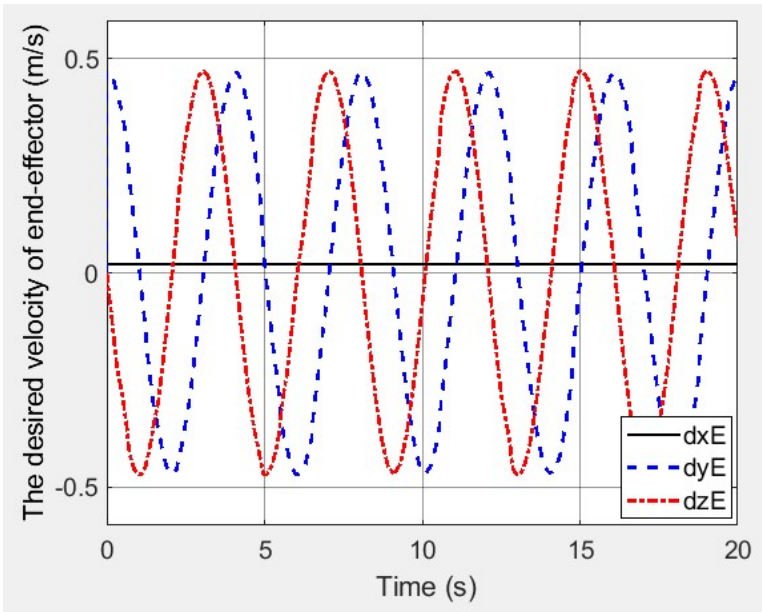

Fig. 6 . The velocity of the end-effector

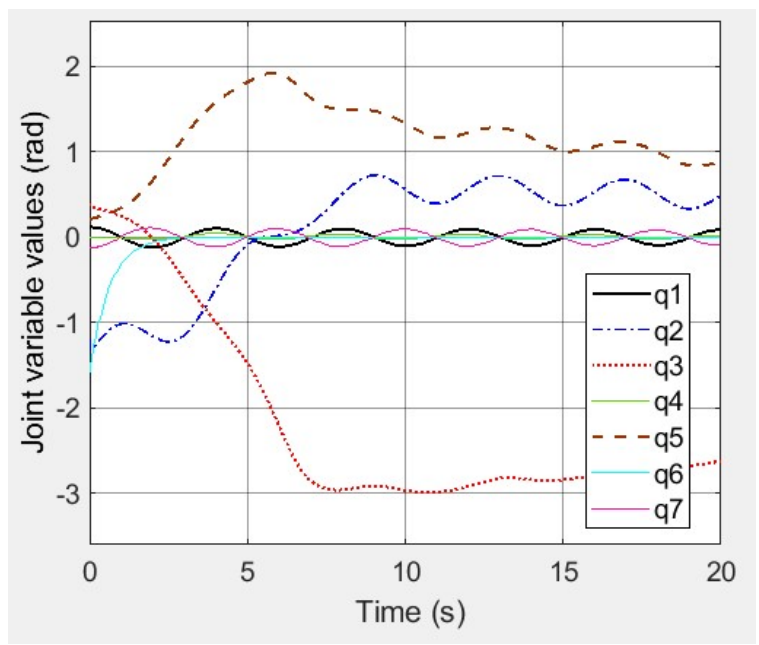

Fig. 7. The joint movements

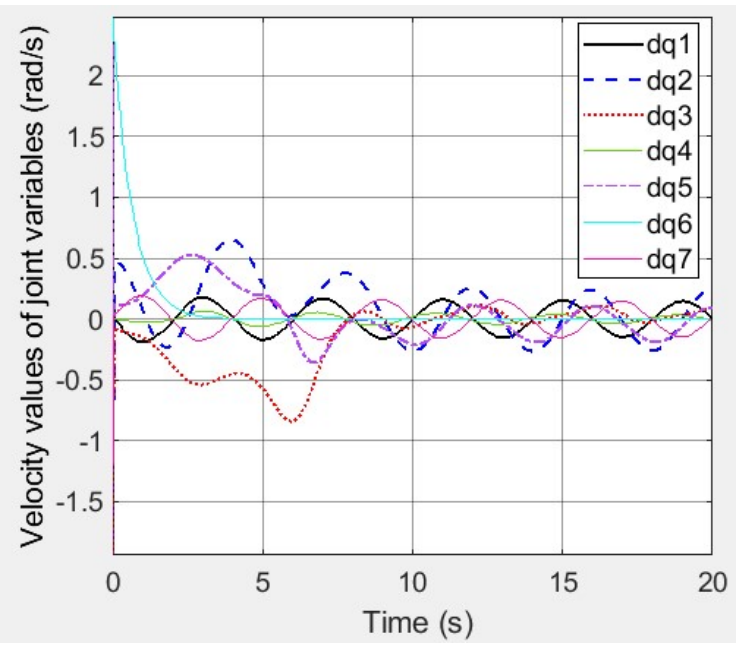

Fig. 8. The joint velocities

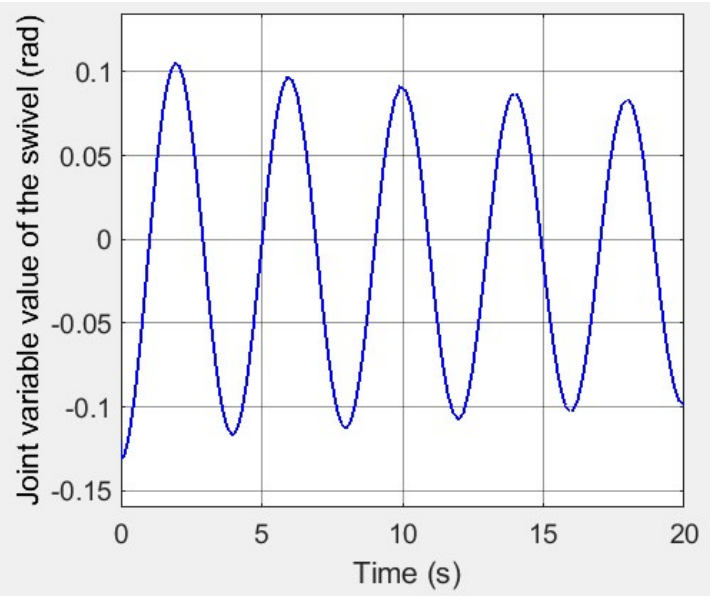

Fig. 9. The rotation of the positioner

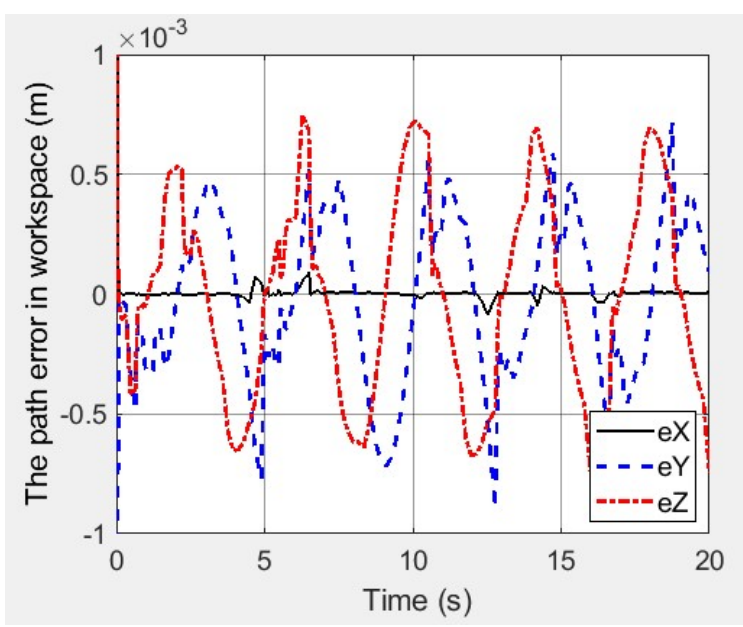

Fig. 10. The path errors

\section{CONCLUSIONS}

A new inverse kinematic algorithm for a welding robot cooperated with a rotary positioner was successfully constructed in this paper. By using the proposed computation law $(\mathrm{Eq}(13))$, the error between the desired welding path and the actual welding path is minimized which can be controlled via the control parameters $\mathbf{K}_{p}$. The numerical example and simulation results show and the robustness of the algorithm and the effectiveness of the proposed method. The maximum value of the tracking error in the example is smaller than $0.001 \mathrm{~mm}$ that demonstrates a the desired accuracy of the kinematics model and the computational results.

Experiments and a complete postprocessor for the robotic system will be the future work of our research.

\section{REFERENCES}

[1] D. Kim, J-S. Choi and B. Nnaji, "Robot arc welding operations planning with a rotating/tilting positioner," International journal of production research, vol. 36, no. 4, pp. 957-979. 1998. https://doi.org/10.1080/002075498193471. 
[2] A. Pashkevich, A. Dolgui and K. Semkin, "Kinematic aspects of a robot-positioner system in an arc welding application," Control Engineering Practice, vol. 11, no. 6, pp. 633-647. 2003.

[3] T. Inoue, K. Nakayama and T. Iwayama, "Positioner for arc welding and arc welding robot system having the positioner," U.S. Patent Application 11/439,964, filed December 14, 2006.

[4] M. Tsai, L. Shi-Da and M. Chen, "Mathematical model for robotic arcwelding off-line programming system," International Journal of Computer Integrated Manufacturing, vol. 5, no. 4-5, pp. 300-309. 1992.

[5] A. Dolgui, A. Pashkevich and K. Semkin, "Manufacturing process planning for robotic arc-welding station with positioning table," In (ISATP 2005). The 6th IEEE International Symposium on Assembly and Task Planning: From Nano to Macro Assembly and Manufacturing, 2005., pp. 1-6., 2005.

[6] J. Gao, A. Pashkevich and S. Caro, "Optimization of the robot and positioner motion in a redundant fiber placement workcell," Mechanism and Machine Theory, vol. 114, pp. 170-189. 2017.

[7] H. C. Fang, S. Ong and A. Nee, "Robot path planning optimization for welding complex joints," The International Journal of Advanced Manufacturing Technology vol. 90, no. 9-12, pp. 3829-3839. 2017.

[8] C.A. My and E.L.J. Bohez, "New algorithm to minimise kinematic tool path errors around 5-axis machining singular points," International Journal of Production Research, vol. 54, no. 20, pp. 5965-75. 2016. https://doi.org/10.1080/00207543.2015.1134838

[9] C.A. My, "Integration of CAM systems into multi-axes computerized numerical control machines," Proceedings of the Second IEEE International Conference on Knowledge and Systems Engineering (KSE), Hanoi, Vietnam, 2010, pp. 119-124. doi: 10.1109/KSE.2010.30

[10] K. Sørby, "Inverse kinematics of five-axis machines near singular configurations," International Journal of Machine Tools and Manufacture, vol. 47, no. 2, pp. 299-306. 2007. https://doi.org/10.1016/j.ijmachtools.2006.03.011

[11] O.R. Tutunea-Fatan and H.Y. Feng, "Configuration analysis of fiveaxis machine tools using a generic kinematic model," International Journal of Machine Tools and Manufacture, vol. 44, no. 11, pp. 123543. 2004. https://doi.org/10.1016/j.ijmachtools.2004.03.009

[12] C.H. She and R.S. Lee, "A postprocessor based on the kinematics model for general five-axis machine tools," Journal of Manufacturing Processes, vol. 2, no. 2, pp. 131-141. 2000.

[13] C.A. My, C. H. Le, M. Packianather and E. Bohez, "Novel robot arm design and implementation for hot forging press automation," International Journal of Production Research, pp. 1-15. 2018. https://doi.org/10.1080/00207543.2018.1521026

[14] C.A. My and L.C. Thanh, "Inverse dynamic of a N-links manipulator mounted on a wheeled mobile robot," Proceedings of the IEEE International Conference on Control, Automation and Information Sciences (ICCAIS), Nha Trang, Vietnam, 2013, pp. 164170. Doi:10.1109/ICCAIS.2013.6720548 